\title{
Bioefficacy of mosquito mat vaporizers and associated metabolic detoxication mechanisms in Aedes aegypti (Linnaeus) in Selangor, Malaysia: A statewide assessment
}

\author{
Azratul-Hizayu, T. ${ }^{1}$, Chen, C.D. ${ }^{2,3}$, Lau, K.W. ${ }^{2}$, Azrizal-Wahid, N. ${ }^{1}$, Tan, T.K. ${ }^{4}$, Lim, Y.A.L. ${ }^{4}$, \\ Sofian-Azirun, M. ${ }^{2}$, Low, V.L. ${ }^{*}$ \\ ${ }^{1}$ Institute for Advanced Studies (IAS), Universiti Malaya, 50603 Kuala Lumpur, Malaysia \\ ${ }^{2}$ Institute of Biological Sciences, Faculty of Science, Universiti Malaya, 50603 Kuala Lumpur, Malaysia \\ ${ }^{3}$ Rimba Ilmu Botanical Garden, Universiti Malaya, 50603 Kuala Lumpur, Malaysia \\ ${ }^{4}$ Department of Parasitology, Faculty of Medicine, Universiti Malaya, 50603 Kuala Lumpur, Malaysia \\ ${ }^{5}$ Higher Institution Centre of Excellence (HICoE), Tropical Infectious Diseases Research \& Education Centre (TIDREC), Universiti Malaya, \\ 50603 Kuala Lumpur, Malaysia \\ *Corresponding author: vanlun_low@um.edu.my
}

\section{ARTICLE HISTORY}

Received: 11 May 2021

Revised: 1 July 2021

Accepted: 1 July 2021

Published: 30 August 2021

\begin{abstract}
This study aims to examine the efficacy of mosquito mat vaporizers on Aedes aegypti and their associated metabolic detoxication mechanisms. For this purpose, Aedes aegypti (Linnaeus) was collected from nine districts in Selangor, Malaysia and tested with mosquito vaporizing mat bioassays. The same populations were also subjected to biochemical assays to investigate activities of detoxifying enzymes, namely non-specific esterase (EST), glutathione-S-transferase (GST) and mixed function oxidase (MFO). The efficacy of Ae. aegypti on the active ingredients tested in decreasing order were $\mathrm{d}$ - allethrin > dimefluthrin > prallethrin with $\mathrm{PBO}>$ prallethrin. The results further indicated significant enhancement mean levels of EST, GST and MFO in pyrethroid-resistant populations. The mortality rate of Ae. aegypti in response to pyrethroid active ingredients was associated with MFO activity, suggesting it is an important detoxification enzyme for the populations tested. In view of the presence of resistance against household insecticide products, pyrethroid efficacy on Ae. aegypti populations needs to be monitored closely to ensure the implementation of an effective vector control program in Malaysia.
\end{abstract}

Keywords: Resistance; mosquito mat vaporizer; biochemical assays; pyrethroid; Aedes.

\section{INTRODUCTION}

Dengue and chikungunya are two major public health issues in Malaysia with 130, 101 severe dengue cases and 990 chikungunya cases reported in 2019 (Ministry of Health Malaysia [MOH], 2020). Aedes aegypti and Ae. albopictus are responsible for the transmissions of dengue and chikungunya viruses in Malaysia. Aedes aegypti is the primary dengue vector which lives close to humans in urban surroundings, whereas Ae. albopictus serves as the secondary dengue vector which mainly lives outdoor (Vontas et al., 2012).

The mosquito control program in Malaysia has been carried out as an integrated program that involves environmental management and source reduction through public education and enforcement. The control program highlights two new features: cross-sector and inter-agency cooperation; and a decision-making support system based on four fundamental aspects, namely cases, viruses, entomological monitoring and ecological information (Ministry of Health
Malaysia $[\mathrm{MOH}], 2009)$. Of these, insecticide application is an one of the important control measures to combat mosquito-borne diseases worldwide including Malaysia. In addition to larviciding and adulticiding activities, household insecticide products containing pyrethroid active ingredients have been widely used worldwide. The efficacy of the commonly used household pyrethroid products against Ae. aegypti, however, has been understudied. Essentially, this work seeks to examine the susceptibility of Ae. aegypti adults to the commercial mosquito mat vaporizers used by the community in Selangor, Malaysia, and attempts to characterize the detoxification mechanisms in pyrethroidresistant populations.

\section{MATERIALS AND METHODS}

Study sites

Aedes aegypti eggs were collected using ovitraps from nine districts: Sabak Bernam, Kuala Selangor, Hulu Selangor, 


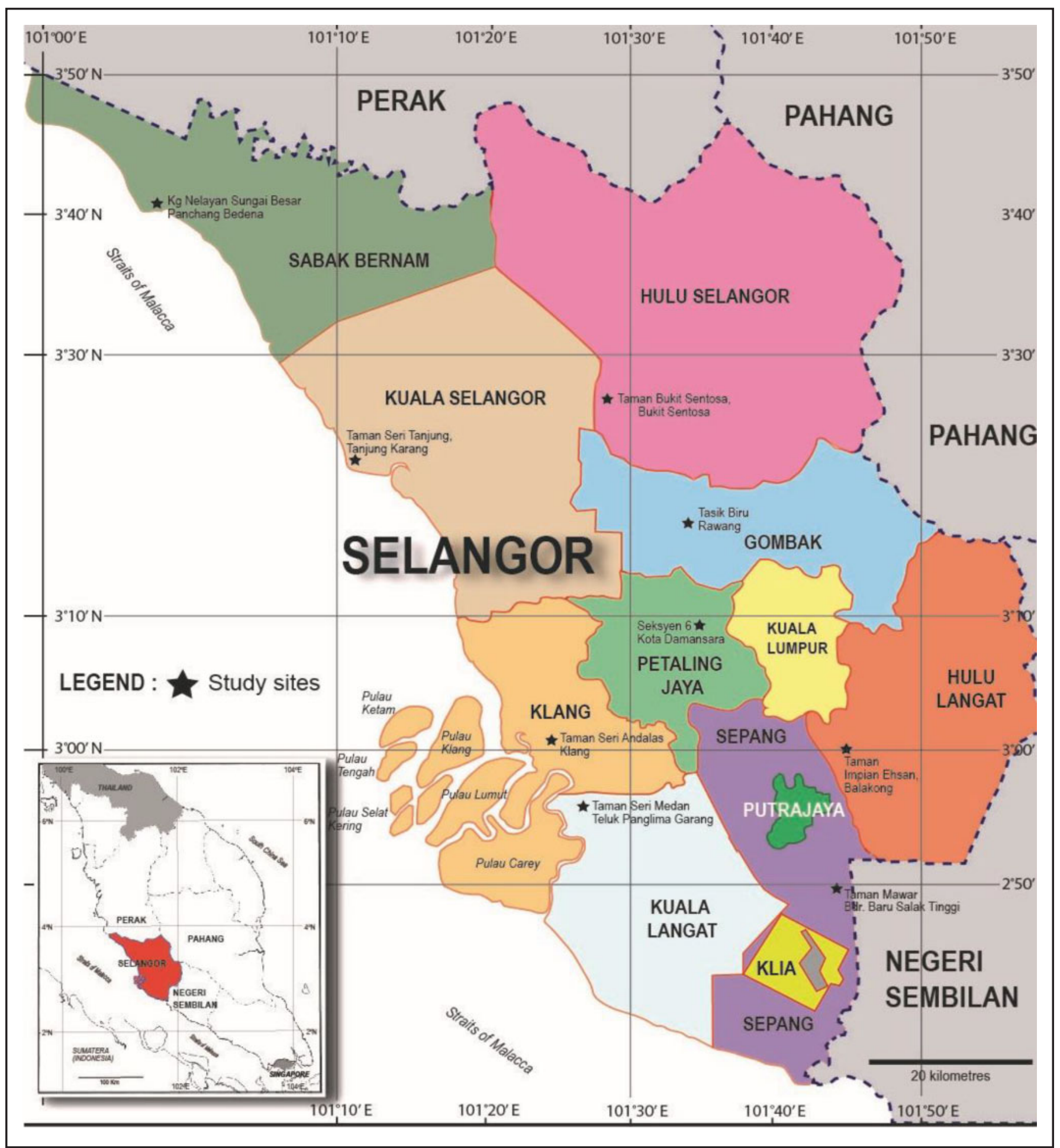

Figure 1. Ovitrap collection sites in Selangor.

Gombak, Petaling, Hulu Langat, Kuala Langat, Klang and Sepang (Figure 1).

\section{Preparation of ovitrap and sample collection}

Ovitraps were used as designed by Lee (1992). The ovitrap consisted of a $300-\mathrm{ml}$ black coloured plastic cup with $9.0 \mathrm{~cm}$ in height, diameter base of $6.5 \mathrm{~cm}$ with an opening of $7.8 \mathrm{~cm}$. Each ovitrap was fixed with a $2.5 \mathrm{~cm} \times 10.0 \mathrm{~cm} \times 0.3 \mathrm{~cm}$ hardboard paddle. The ovitrap was then filled up with 5.5 $\mathrm{cm}$ of chlorine-free tap water. For each study site, 40 ovitraps were placed randomly in close proximity with other potential larval habitats which were protected against direct sunlight and rain. After five days, the ovitraps were collected and transported to the laboratory for hatching, rearing and subsequent identification of adult phase.

\section{Colonization of Aedes aegypti}

Aedes aegypti was identified and colonized according to locations in respective wooden built, and net covered cages $(30 \mathrm{~cm} \times 30 \mathrm{~cm} \times 30 \mathrm{~cm})$. The adult mosquitoes were fed with $10 \%$ sucrose solution as their food source. Female adults aged 4-5 days were fed with blood meal using a white mouse until full engorgement. An oviposition site consisted of a plastic cup with $200 \mathrm{ml}$ chlorine-free water lined with No 1 
Whatman filtered paper and placed into a cage after two days of blood feeding. The eggs were left to hatch with chlorine-free water-filled in plastic containers $(25 \mathrm{~cm} \times 30$ $\mathrm{cm} \times 5 \mathrm{~cm}$ ). Larvae were fed with powdered beef liver. Pupae were then placed in a small plastic cup and put into rearing cage to grow as adults. The Ae. aegypti Bora-Bora strain obtained from the Universiti Sains Malaysia, same as those in Amelia-Yap et al. (2018a, 2019), was used as the susceptible reference population.

\section{Mosquito vaporizing mat bioassay}

Four commercial mosquito mat vaporizers, prallethrin $15.0 \mathrm{mg} / \mathrm{mat}$ with piperonyl butoxide [PBO] $18.0 \mathrm{mg} / \mathrm{mat}$, dimefluthrin $7.4 \mathrm{mg} / \mathrm{mat}$, prallethrin $15.0 \mathrm{mg} / \mathrm{mat}$ and $\mathrm{d}$ allethrin $40.0 \mathrm{mg} / \mathrm{mat}$ were used in the present study. The bioassays were performed using the standardized protocol defined by the World Health Organization [WHO] (2009), and the World Health Organization [WHO] (2016) resistance indicator was adopted. Transparent glass chambers $(70 \mathrm{~cm} \times$ $70 \mathrm{~cm} \times 70 \mathrm{~cm})$ that included a sliding window $(18 \mathrm{~cm} \times 20 \mathrm{~cm})$ were used for bioassays. Temperatures and relative humidity were maintained at $27 \pm 2^{\circ} \mathrm{C}$ and $80 \pm 10 \%$ for the duration of bioassays.

Mosquito mat was inserted into its vaporizing device and was heated outside the test chamber. At the intended test intervals, the device was introduced into the centre of the glass chamber and allowed to operate continuously. At suitable intervals, the amount of knocked down specimens were observed for 60 minutes. In total, twenty-five, 2 to 5 -dold sugar-fed Ae. aegypti females were released into the chamber and exposed to the mats. The number of knockeddown mosquitoes was calculated and documented per minute, up to 60 minutes. Mosquitoes that were unable to fly or in imbalance posture would be considered as a knockdown. After 60 minutes of exposure time, tested mosquitoes were transferred into a clean plastic container size $9.0 \mathrm{~cm}$ in height, diameter base of $6.5 \mathrm{~cm}$ with an opening of $7.8 \mathrm{~cm}$ using an electric aspirator and held for 24-h postexposure observation. Containers were covered with a mesh and mosquitoes were provided a $10 \%$ sucrose solution via a soaked cotton wool. Mosquitoes were maintained at $27 \pm$ $2{ }^{\circ} \mathrm{C}$ and relative humidity of $80 \pm 10 \%$. Mortality readings were taken 24 -h after mosquitoes had been removed from vapor exposure. Following the mortality reading, dead and alive mosquitoes were transferred to individual microfuge tubes and stored at $-20^{\circ} \mathrm{C}$.

Before subsequent test, the chamber was cleaned with detergent and water. For control experiments, 25 female mosquitoes were released in the cleaned chamber for 60 minutes to avoid any insecticide contamination after cleaning without exposing them to any mats. For each study location and active ingredient, toxicological tests were conducted in three replicates.

\section{Enzyme assays}

For each of the three enzyme assays, 24 individual Ae. aegypti females from each location were used for a total of 720 individuals assayed. The non-specific esterase (EST) enzyme assay was carried out according to the protocol by Brogdon et al. (1988) and Lee (1990). A total of 24 single mosquitoes were homogenized and centrifuged at $4^{\circ} \mathrm{C}$ in phosphatebuffered solutions for 10 minutes at $15,000 \mathrm{rpm}$. This assay then obtained four supernatant aliquots $(50 \mu \mathrm{l})$ derived from single mosquito homogeneity. In a 96 -well plate, a $50 \mu \mathrm{l}$ of indicator (fast blue B salt) was placed on substrate solution (either $\alpha$-naphthyl acetate or $\beta$-naphthyl acetate) and left up to one minute. After an incubation period of 10 minutes, $50 \mu \mathrm{l} 10 \%$ acetic acid was added to stop the reaction. An absorbance reader for the optical dense (BIO-TEK ELX800) was used to measure the density of $450 \mathrm{~nm}$.

Glutathione-S-transferase (GST) enzyme assay was performed according to the protocol by Lee \& Chong (1995). In the potassium phosphate buffer solution, 24 individual mosquitoes were homogenized. Subsequently, centrifugation was conducted at $14,000 \mathrm{rpm}$ at $4^{\circ} \mathrm{C}$ for 10 minutes. Four homogeneous aliquots (each $50 \mu \mathrm{l}$ ) from each mosquito were added in a 96-well plate, followed by the addition of $50 \mu \mathrm{l}$ of $2-\mathrm{mm}$ glutathione and $50 \mu \mathrm{l} 1 \mathrm{mM}$ of 1-chloro-2,4dinitrobenzene. The reaction was incubated within 30 minutes. The data was then recorded at $410 \mathrm{~nm}$ of the optical density.

The mixed function oxidases (MFO) enzyme assay was conducted based on the method by Brogdon et al. (1997). A total of 24 individual mosquitoes were homogenized in a sodium acetate buffer solution. Four homogeneous aliquots $(100 \mu \mathrm{l})$ were obtained from all specimens. After 5-minutes incubation, absorption was determined at $630 \mathrm{~nm}$ with the

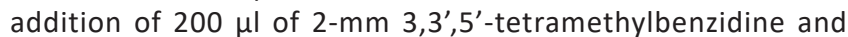
$25 \mu \mathrm{l}$ of $3 \%$ hydrogen peroxide.

\section{Data analysis}

Bioassay data from at least three mosquito mat vaporizer replicates were collected and analyzed. Time to knockdown $\left(\mathrm{KT}_{50}\right)$ was calculated by using probit analysis with SPSS software (version 20) (Finney, 1971). Resistance ratios were calculated using the following formula from

$$
R R=\frac{K T_{50} \text { of field strain }}{K T_{50} \text { of reference strain }}
$$

RR values of $<5$ imply low resistance, 5-10 imply medium resistance, while $>10$ imply high resistance (Mazzarri \& Georghiou, 1995). A one-way variance analysis (ANOVA) was performed using SPSS Version 20 to compare the knockdown and mortality rates in all study sites. Tukey's test used to determine the mean for significant ANOVAs, $P<0.05$. In order to examine the presence of cross-resistance of the active ingredient tested, Spearman's rank-order correlation analysis between knockdown rates was performed (Bisset et al., 1997). To assess mosquito susceptibility, the mortality rate after 24-h post-treatment was recorded (WHO, 2016).

- Mortality rate of $\geq 98-100 \%$ : susceptible to insecticide

- Mortality rate of $<98 \%$ : possible development of resistance to insecticide

- Mortality rate of $<90 \%$ : resistance to insecticide

The Spearman rank-order correlation analysis was correlated with the mortality rate of mosquito mat vaporizing bioassays tested on 24 samples per test with triplicates of each population. The ratio of enzyme activity was determined by dividing the mean enzyme level of the field strain, and the mean enzyme level of the laboratory reference strain. Using SPSS version 20, a one-way variance analysis (ANOVA) was run to compare mean enzyme activity between study sites. The Tukey test was used to determine the mean for ANOVAs, $P<0.05$. An independent-sample t-test was performed to show any differences in the mean of enzyme activity. 


\section{RESULTS}

\section{Mosquito vaporizing mat bioassay}

Aedes aegypti populations tested exhibited different trends in susceptibility to pyrethroid active ingredients. BoraBora laboratory reference that was tested with mosquito mat vaporizer resulted in $100 \%$ mortality in all replicates, with $\mathrm{KT}_{50} 0.39$ minutes to prallethrin with $\mathrm{PBO}, 1.35$ minutes to dimefluthrin, 0.91 minutes to prallethrin and 0.38 minutes to $d$-allethrin. The $\mathrm{KT}_{50}$ of field population exposed to prallethrin with $\mathrm{PBO}$, dimefluthrin, prallethrin and d-allethrin ranged from 2.56 to 13.06 minutes (the longest $\mathrm{KT}_{50}$ population: Hulu Selangor); 1.44 to 4.41 minutes (the longest $\mathrm{KT}_{50}$ population: Hulu Selangor); 6.58 to 37.07 minutes (the longest $\mathrm{KT}_{50}$ population: Hulu Langat) and 2.72 to 23.46 minutes (the longest $\mathrm{KT}_{50}$ population: Kuala Langat) (Table 1).

Aedes aegypti populations demonstrated different percentages of knockdown from 80.00 to $98.67 \%$ (the lowest knockdown rates: Hulu Langat population), 96.00 to $100 \%$ (the lowest knockdown rates: Petaling population), 50.67 to 90.67\% (the lowest knockdown rates: Hulu Langat population) and 76.00 to $100.00 \%$ (the lowest knockdown rates: Kuala Langat population) for prallethrin with $\mathrm{PBO}$, dimefluthrin, prallethrin and d-allethrin, respectively (Table 2 ).

Mortality was observed after exposure to prallethrin with PBO, dimefluthrin, prallethrin and d-allethrin, respectively in Ae. aegypti populations ranging from 69.33-100\%, 73.33$100 \%, 72-97.33 \%$ and $85.33-100 \%$. Populations from Kuala Selangor, Gombak, Petaling and Sepang showed high susceptibility to d-allethrin with $100 \%$ mortality at the end 24-hr reading. Meanwhile, the population from Sabak Bernam, Kuala Selangor, Hulu Langat and Kuala Langat showed $<90 \%$ mortality, suggesting that they were resistant to prallethrin. Spearman rank analysis showed significant correlations between prallethrin with $\mathrm{PBO}$ and dimefluthrin mortality rates $(r=0.828 ; P=0.003)$, prallethrin with $\mathrm{PBO}$ prallethrin and d-allethrin $(r=0.839 ; P=0.002)$ as well as dimefluthrin and d-allethrin $(r=0.822 ; P=0.004)$.

\section{Enzyme assays}

Non-specific esterases (EST) assay demonstrated enzyme ratios ranging from 1.00 to 2.07 fold for $\alpha$-esterases activity and from 1.00 to 2.08 fold for $\beta$-esterases activity. Activities of $\alpha$-esterases and $\beta$-esterases had significantly increased in all populations except Hulu Selangor and Klang. All populations at nine sites showed higher $\alpha$-esterase activity compared to $\beta$-esterase activity, except for Kuala Selangor, Kuala Langat and Klang populations. The ratios of glutathione-S-transferase (GST) ranged from 1.14 to 1.71 folds were recorded. Seven populations (i.e., Sabak Bernam, Kuala Selangor, Hulu Selangor, Hulu Langat, Kuala Langat, Klang and Sepang) showed a significant increase of glutathioneS-transferase activity. Slightly elevated of mixed function oxidases (MFO) activity was found in all populations (except Petaling) with ratios ranging from 1.19 to 3.76 folds. Furthermore, one way ANOVA showed that the mean for all enzyme activity tested in Ae. aegypti was significantly different across all study sites $(P<0.001)$ (Table 3$)$.

A significant correlation between prallethrin and $\mathrm{PBO}$ survivability rate and GST $(r=-0.683 ; P=0.030)$ and prallethrin survivability rate and GST $(r=-0.642 ; P=0.045)$ were recorded

Table 1. $\mathrm{KT}_{50}$ and resistance ratio (RR) of Aedes aegypti adults against prallethrin $15.0 \mathrm{mg} / \mathrm{mat}$ with piperonyl butoxide 18.0 mg/mat, dimefluthrin 7.4 $\mathrm{mg} / \mathrm{mat}$, prallethrin $15.0 \mathrm{mg} / \mathrm{mat}$ and $\mathrm{d}$ - allethrin $40.0 \mathrm{mg} / \mathrm{mat}$

\begin{tabular}{|c|c|c|c|c|c|c|c|c|}
\hline \multirow{3}{*}{ Strain } & \multicolumn{8}{|c|}{ Active ingredients } \\
\hline & \multicolumn{2}{|c|}{$\begin{array}{l}\text { prallethrin with } \\
\text { piperonyl butoxide }\end{array}$} & \multicolumn{2}{|c|}{ dimefluthrin } & \multicolumn{2}{|c|}{ prallethrin } & \multicolumn{2}{|c|}{ d- allethrin } \\
\hline & $\begin{array}{c}\mathrm{KT}_{50}(\min ) \\
(95 \% \mathrm{CL})\end{array}$ & $\mathrm{RR}$ & $\begin{array}{c}\mathrm{KT}_{50}(\mathrm{~min}) \\
(95 \% \mathrm{CL})\end{array}$ & $\mathrm{RR}$ & $\begin{array}{c}\mathrm{KT}_{50}(\min ) \\
(95 \% \mathrm{CL})\end{array}$ & $\mathrm{RR}$ & $\begin{array}{c}\mathrm{KT}_{50}(\min ) \\
(95 \% \mathrm{CL})\end{array}$ & $\mathrm{RR}$ \\
\hline Reference & $\begin{array}{c}0.39 \\
(0.34-0.44)\end{array}$ & - & $\begin{array}{c}1.35 \\
(0.86-1.88)\end{array}$ & - & $\begin{array}{c}0.91 \\
(0.80-1.03)\end{array}$ & - & $\begin{array}{c}0.38 \\
(0.33-0.43)\end{array}$ & - \\
\hline Sabak Bernam & $\begin{array}{c}4.39 \\
(3.83-4.95)\end{array}$ & 11.26 & $\begin{array}{c}3.17 \\
(2.84-3.47)\end{array}$ & 2.35 & $\begin{array}{c}10.77 \\
(9.70-11.81)\end{array}$ & 11.84 & $\begin{array}{c}4.22 \\
(3.46-4.98)\end{array}$ & 11.11 \\
\hline Kuala Selangor & $\begin{array}{c}7.37 \\
(6.61-8.13)\end{array}$ & 18.90 & $\begin{array}{c}2.01 \\
(1.59-2.41)\end{array}$ & 1.49 & $\begin{array}{c}7.61 \\
(6.73-8.50)\end{array}$ & 8.36 & $\begin{array}{c}6.63 \\
(5.89-7.37)\end{array}$ & 17.45 \\
\hline Hulu Selangor & $\begin{array}{c}13.06 \\
(11.71-14.35)\end{array}$ & 33.49 & $\begin{array}{c}4.41 \\
(4.09-4.70)\end{array}$ & 3.27 & $\begin{array}{c}6.58 \\
(5.94-7.22)\end{array}$ & 7.23 & $\begin{array}{c}8.93 \\
(8.01-9.83)\end{array}$ & 23.50 \\
\hline Gombak & $\begin{array}{c}6.62 \\
(5.81-7.41)\end{array}$ & 16.97 & $\begin{array}{c}3.05 \\
(2.06-3.84)\end{array}$ & 2.26 & $\begin{array}{c}27.67 \\
(25.28-30.34)\end{array}$ & 30.41 & $\begin{array}{c}8.29 \\
(7.45-9.11)\end{array}$ & 21.82 \\
\hline Petaling & $\begin{array}{c}4.97 \\
(4.43-5.51)\end{array}$ & 12.74 & $\begin{array}{c}6.02 \\
(5.42-6.62)\end{array}$ & 4.46 & $\begin{array}{c}10.30 \\
(9.59-10.99)\end{array}$ & 11.32 & $\begin{array}{c}4.05 \\
(3.52-4.61)\end{array}$ & 10.66 \\
\hline Hulu Langat & $\begin{array}{c}5.35 \\
(4.03-6.65)\end{array}$ & 13.72 & $\begin{array}{c}3.11 \\
(2.88-3.34)\end{array}$ & 2.30 & $\begin{array}{c}37.07 \\
(33.63-41.32)\end{array}$ & 40.74 & $\begin{array}{c}14.20 \\
(12.88-15.50)\end{array}$ & 37.37 \\
\hline Kuala Langat & $\begin{array}{c}7.62 \\
(6.79-8.46)\end{array}$ & 19.54 & $\begin{array}{c}2.50 \\
(2.28-2.70)\end{array}$ & 1.85 & $\begin{array}{c}31.55 \\
(29.83-33.44)\end{array}$ & 34.67 & $\begin{array}{c}23.46 \\
(21.78-25.21)\end{array}$ & 61.74 \\
\hline Klang & $\begin{array}{c}2.56 \\
(2.17-2.95)\end{array}$ & 6.56 & $\begin{array}{c}1.44 \\
(1.31-1.57)\end{array}$ & 1.07 & $\begin{array}{c}8.44 \\
(7.27-9.57)\end{array}$ & 9.27 & $\begin{array}{c}2.72 \\
(1.85-3.63)\end{array}$ & 7.16 \\
\hline Sepang & $\begin{array}{c}3.19 \\
(2.72-3.64)\end{array}$ & 8.18 & $\begin{array}{c}2.67 \\
(2.43-2.91)\end{array}$ & 1.98 & $\begin{array}{c}8.81 \\
(7.96-9.64)\end{array}$ & 9.68 & $\begin{array}{c}3.38 \\
(3.16-3.80)\end{array}$ & 8.89 \\
\hline
\end{tabular}

$\mathrm{CL}$ - confidence limit. $\mathrm{CL}$ does not overlap with the reference strain are significantly different from the reference strain. 
Table 2. Percentages of knockdown and mortality of Aedes aegypti adults against prallethrin $15.0 \mathrm{mg} / \mathrm{mat}$ with piperonyl butoxide $18.0 \mathrm{mg} / \mathrm{mat}$, dimefluthrin $7.4 \mathrm{mg} / \mathrm{mat}$, prallethrin $15.0 \mathrm{mg} / \mathrm{mat}$ and d- allethrin $40.0 \mathrm{mg} / \mathrm{mat}$

\begin{tabular}{|c|c|c|c|c|c|c|c|c|}
\hline \multirow{3}{*}{ Strain } & \multicolumn{4}{|c|}{ Knockdown } & \multicolumn{4}{|c|}{ Mortality } \\
\hline & $\begin{array}{l}\text { prallethrin } \\
\text { with piperonyl } \\
\text { butoxide }\end{array}$ & dimefluthrin & prallethrin & d- allethrin & $\begin{array}{l}\text { prallethrin } \\
\text { with piperonyl } \\
\text { butoxide }\end{array}$ & dimefluthrin & prallethrin & d- allethrin \\
\hline & $\begin{array}{c}15.0 \mathrm{mg} / \mathrm{mat} \\
\text { with } \\
18.0 \mathrm{mg} / \mathrm{mat}\end{array}$ & $7.4 \mathrm{mg} / \mathrm{mat}$ & $15.0 \mathrm{mg} / \mathrm{mat}$ & $40.0 \mathrm{mg} / \mathrm{mat}$ & $\begin{array}{c}15.0 \mathrm{mg} / \mathrm{mat} \\
\text { with } \\
18.0 \mathrm{mg} / \mathrm{mat}\end{array}$ & 7.4 mg/mat & $15.0 \mathrm{mg} / \mathrm{mat}$ & $40.0 \mathrm{mg} / \mathrm{mat}$ \\
\hline Reference & 100.00 & 100.00 & 100.00 & 100.00 & 100.00 & 100.00 & 100.00 & 100.00 \\
\hline Sabak Bernam & 96.00 & $100.00^{a}$ & 81.33 & 92.00 & ${ }^{R} 88.00$ & 92.00 & R 72.00 & 92.00 \\
\hline Kuala Selangor & 89.33 & $100.00^{b}$ & 84.00 & 89.33 & 93.33 & 93.33 & R 74.67 & 100.00 \\
\hline Hulu Selangor & 84.00 & $100.00^{c}$ & 89.33 & 82.67 & ${ }^{R} 80.00$ a & $\mathrm{R} 73.33^{\mathrm{a}}$ & 97.33 & 92.00 \\
\hline Gombak & 90.67 & $100.00^{d}$ & 58.67 & 92.00 & 96.00 & 92.00 & 96.00 & 100.00 \\
\hline Petaling & 92.00 & 96.00 abcdefgh & 90.67 & 96.00 & 100.00 & 93.33 & 96.00 & 100.00 \\
\hline Hulu Langat & 80.00 & $100.00 \mathrm{~g}$ & 50.67 & 84.00 & 90.67 & R 88.00 & R 76.00 & 96.00 \\
\hline Kuala Langat & 84.00 & $100.00^{f}$ & 72.00 & 76.00 & R $69.33^{a}$ & R 89.33 & R76.00 & 94.67 \\
\hline Klang & 97.33 & 100.00 e & 81.33 & 85.33 & R 81.33 & R 86.67 & 92.00 & R 85.33 \\
\hline Sepang & 98.67 & $100.00^{h}$ & 86.67 & 100.00 & 97.33 & $100.00^{a}$ & 96.00 & 100.00 \\
\hline \multirow[t]{2}{*}{ One-way ANOVA } & $P=0.421$ & $P=0.020$ & $P=0.097$ & $P=0.448$ & $P=0.010$ & $P=0.020$ & $P=0.067$ & $P=0.580$ \\
\hline & $\begin{aligned} F & =1.076 \\
d f & =(9,20)\end{aligned}$ & $\begin{aligned} F & =3.000 \\
d f & =(9,20)\end{aligned}$ & $\begin{array}{l}F=1.984 \\
d f=(9,20)\end{array}$ & $\begin{aligned} F & =1.035 \\
d f & =(9,20)\end{aligned}$ & $\begin{aligned} F & =4.465 \\
d f & =(9,20)\end{aligned}$ & $\begin{array}{l}F=2.214 \\
d f=(9,20)\end{array}$ & $\begin{aligned} F & =2.994 \\
d f & =(9,20)\end{aligned}$ & $\begin{aligned} F & =0.852 \\
d f & =(9,20)\end{aligned}$ \\
\hline
\end{tabular}

Means followed by a different letter were significantly different, $P<0.05$, Tukey's test. $\mathrm{R}=$ resistant (mortality $<90 \%$ ) and $\mathrm{S}=$ susceptible (mortality $>98 \%$ ) as determined by WHO (2016). Knockdown rate was determined after 60-min exposure; mortality was calculated 24 h post-exposure.

Table 3. Mean ( \pm SE) level of non-specific esterases ( $\alpha$-and $\beta$-EST), glutathione-S-transferase (GST) and mixed function oxidases (MFO) activities of Aedes aegypti sampled from different localities in Selangor

\begin{tabular}{|c|c|c|c|c|c|c|c|c|}
\hline \multirow{2}{*}{ Strain } & \multicolumn{2}{|l|}{$\alpha$-ESTs } & \multicolumn{2}{|l|}{$\beta$-ESTs } & \multicolumn{2}{|l|}{ GSTs } & \multicolumn{2}{|l|}{ MFOs } \\
\hline & $\begin{array}{c}(\alpha-\mathrm{Na} \mathrm{çmol} / \mathrm{min} / \\
\mathrm{mg} \text { protein) }\end{array}$ & ER & $\begin{array}{c}\text { ( } \beta \text {-Na çmol/ } \\
\mathrm{min} / \mathrm{mg} \text { protein) }\end{array}$ & ER & $\begin{array}{l}\text { (CDNA-çmol/min } \\
\text { /mg protein) }\end{array}$ & ER & $\begin{array}{c}\text { (Absorbance } \\
630 \mathrm{~nm} \text { ) }\end{array}$ & ER \\
\hline Reference & $0.14 \pm 0.01$ & - & $0.13 \pm 0.02$ & - & $0.07 \pm 0.01$ & - & $0.21 \pm 0.04$ & - \\
\hline Sabak Bernam & $* 0.22 \pm 0.01 \mathrm{gkpqr}$ & 1.57 & $* 0.21 \pm 0.01 \mathrm{fk}$ & 1.62 & $* 0.11 \pm 0.00$ bekno & 1.57 & $* 0.35 \pm 0.02$ dpst & 1.67 \\
\hline Kuala Selangor & $* 0.20 \pm 0.01 \mathrm{abc}$ & 1.43 & $* 0.20 \pm 0.01 \mathrm{ab}$ & 1.54 & $* 0.09 \pm 0.00$ abcd & 1.29 & $* 0.79 \pm 0.03$ abcdefgh & 3.76 \\
\hline Hulu Selangor & $0.14 \pm 0.00$ cfimqs & 1.00 & $0.13 \pm 0.00$ behk & 1.00 & $* 0.09 \pm 0.00$ inps & 1.29 & $* 0.37 \pm 0.01$ fquwx & 1.76 \\
\hline Gombak & $* 0.29 \pm 0.03$ beklmno & 2.07 & $* 0.27 \pm 0.03$ acdfghij & 2.08 & $0.08 \pm 0.00 \mathrm{hklm}$ & 1.14 & $* 0.50 \pm 0.02$ cimpar & 2.38 \\
\hline Petaling & ${ }^{*} 0.22 \pm 0.01$ nst & 1.57 & ${ }^{*} 0.18 \pm 0.01$ & 1.38 & $0.08 \pm 0.00$ dgjogst & 1.14 & $0.25 \pm 0.02$ gkrvwy & 1.19 \\
\hline Hulu Langat & $* 0.20 \pm 0.01$ def & 1.43 & $* 0.17 \pm 0.00^{c}$ & 1.31 & $* 0.09 \pm 0.00$ efg & 1.29 & $* 0.37 \pm 0.05$ aijkl & 1.76 \\
\hline Kuala Langat & $* 0.17 \pm 0.00 \mathrm{hl}$ & 1.21 & $* 0.17 \pm 0.01 \mathrm{~g}$ & 1.31 & $* 0.12 \pm 0.00$ cflpqr & 1.71 & $* 0.52 \pm 0.02$ ejnsuv & 2.48 \\
\hline Klang & $0.16 \pm 0.00$ jort & 1.14 & $0.16 \pm 0.01^{j}$ & 1.23 & $* 0.10 \pm 0.00 \mathrm{mrt}$ & 1.43 & $* 0.53 \pm 0.01$ hlotxy & 2.52 \\
\hline Sepang & $* 0.27 \pm 0.01$ adghijp & 1.93 & $* 0.21 \pm 0.01$ de & 1.62 & $* 0.10 \pm 0.00$ ahij & 1.43 & $* 0.32 \pm 0.01$ bmno & 1.52 \\
\hline
\end{tabular}

$\mathrm{SE}=$ standard error; $\mathrm{ER}=$ enzyme ratio. Mean followed by a different letter were significantly different, $P<0.05$, Tukey's test.

*Significant increase in mean differences compared to the laboratory reference strain, $P<0.05$, t-test.

(Table 4). Besides, an association between $\alpha$-esterase and $\beta$-esterase activity $(r=0.927 ; P=0.0001)$ was also determined (Figure 2.). Table 5 shows a summary of insecticide resistance and the detoxification mechanism in various populations of Ae aegypti. Increased levels of all enzyme activities were found in five populations (i.e., Sabak Bernam, Kuala Selangor, Hulu Langat, Kuala Langat, and Sepang).

\section{DISCUSSION}

Dengue prevention and control largely depend on insecticidebased strategies. Previous studies showed that Ae. aegypti populations in Malaysia were resistant to pyrethroids. Notably, these Ae. aegypti populations were unrelenting in demonstrating the endless evolution of resistance to a wide variety of pyrethroids. The 10 -year studies suggested that a specific class of insecticides remains the cornerstone of the mosquito control program. Resistance identification in Ae. aegypti populations were found to be consistent with the vast majority of findings from previous studies on mat vaporizer (Chadwick \& Lord, 1977; Yap et al., 1995; Adanan et al., 2005;) and mosquito coil (Jantan et al., 1999; Liu et al., 2003; El-garj et al., 2015; Chin et al., 2017; Amelia-Yap et al., 2018a).

Prolonged use of pyrethroids on Ae. aegypti has resulted in the occurrence of pyrethroid resistance. The use of rapidacting insecticides for vector control may confer a high selection pressure which could support the survivability of resistant mosquitoes (Chin et al., 2017; Amelia-Yap et al., 2018a). In this study, most Ae. aegypti showed their recovery 
Table 4. Spearman's rank order correlation between survivability rates in pyrethroid adult bioassays against nonspecific estrease ( $\alpha$ - and $\beta$-esterases), glutathione-S-tranferase (GST) and mixed function oxidase (MFO) activities in different Aedes aegypti populations in Selangor

\begin{tabular}{|c|c|c|c|c|c|c|c|c|}
\hline \multirow{3}{*}{ Strain } & \multicolumn{8}{|c|}{ Active ingredients } \\
\hline & \multicolumn{2}{|c|}{$\begin{array}{l}\text { prallethrin with } \\
\text { piperonyl butoxide }\end{array}$} & \multicolumn{2}{|c|}{ dimefluthrin } & \multicolumn{2}{|c|}{ prallethrin } & \multicolumn{2}{|c|}{ d-allethrin } \\
\hline & r & $P$ & r & $P$ & $r$ & $\mathrm{P}$ & r & $\mathrm{P}$ \\
\hline$\alpha$-esterases & 0.365 & 0.300 & 0.312 & 0.380 & -0.228 & 0.527 & 0.428 & 0.217 \\
\hline$\beta$-esterases & 0.291 & 0.415 & 0.372 & 0.289 & -0.415 & 0.233 & 0.398 & 0.254 \\
\hline GSTs & -0.683 & 0.030 & -0.316 & 0.374 & -0.642 & 0.045 & -0.569 & 0.086 \\
\hline MFOs & -0.608 & 0.062 & 0.502 & 0.140 & -0.560 & 0.092 & -0.324 & 0.360 \\
\hline
\end{tabular}

to all types of mosquito mat vaporizers at 24-h post-exposure. In natural settings, the mosquito mat vaporizer possibly acts as a spatial repellent that inhibits insects rather than kills them (Kawada, 2009; Chin et al., 2017). This observation may occur owing to the high frequency of dengue vector control in Malaysia. The ability of wild mosquito populations to become resistant to insecticides containing pyrethroid were documented elsewhere (Chin et al., 2017; Amelia-Yap et al., 2018b; Sathantriphop et al., 2019; Sayono et al., 2019).

In this study, Ae. aegypti collected from different study sites displayed varying patterns of resistance to the four active ingredients of the pyrethroid evaluated. The increased resistance degree of Ae. aegypti to pyrethroid at the study sites were predictable because the samples were collected from dengue hotspot areas in which dengue control is highly dependent on pyrethroid insecticides. The observation has been documented in various countries, e.g., Malaysia (Ishak et al., 2017; Leong et al., 2018; Rasli et al., 2018), Thailand (Pethuan et al., 2007; Katsuda et al., 2008; Chuaycharoensuk et al., 2011; Chareonviriyaphap et al., 2013, Sathantriphop et al., 2020), Singapore (Koou et al., 2014a, 2014b; Lee et al., 2014) and Indonesia (Hamid et al., 2017, 2018; Haziqah-Rashid et al., 2019; Triana et al., 2019). Likewise, a state level study on Ae. aegypti showed that these populations were highly resistant to mosquito coils containing pyrethroids with resistance ratios up to 122.87 (Chin et al., 2017).

Following a 24-h post-treatment period, gradual recovery was observed in the insecticidal-free setting, suggesting that the knockdown impact could be a transient effect for the species. The variance between the percentage of knockdown and mortality rate might suggest knockdown resistance where the insecticide pressure is removed and recovery is observed. Mosquito mat vaporizer might function as a space repellent to inhibit within insecticide radius, rather than kill (Bibbs \& Kaufman, 2017). However, the impact of other insecticide use on certain pests such as houseflies (Bong \& Zairi, 2010) and cockroaches (Lee et al., 1996) should not be disregarded.

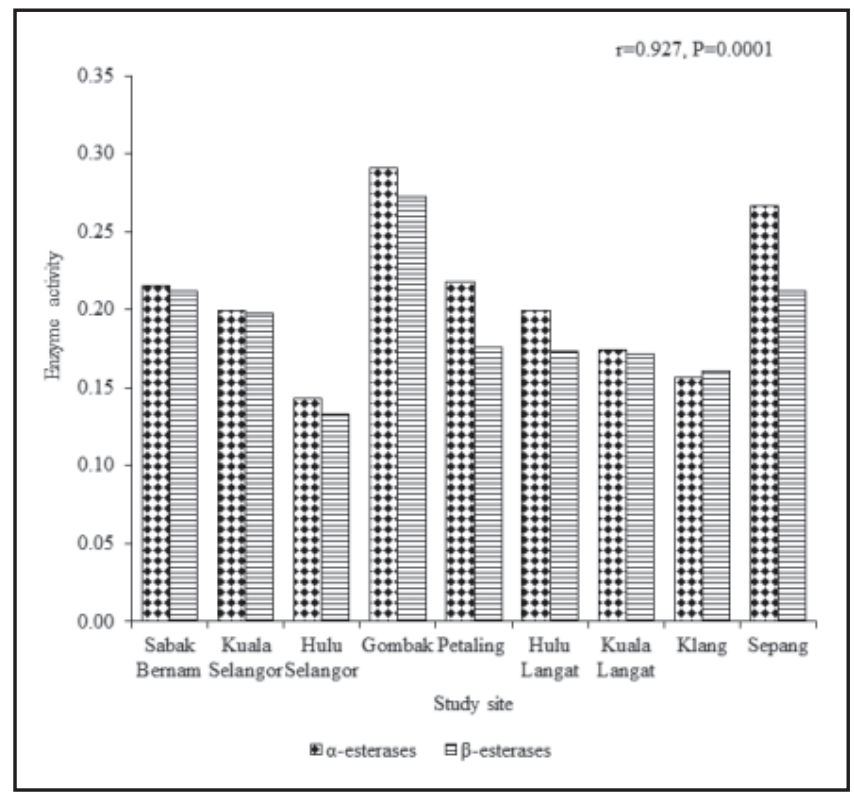

Figure 2. Spearman rank-order correlation between the activity of $\alpha$-esterases and $\beta$-esterases in Aedes aegypti.

Table 5. Summary of insecticide susceptibility and prevalence of resistance mechanisms in different Aedes aegypti populations in Selangor

\begin{tabular}{|c|c|c|c|c|c|c|c|c|}
\hline \multirow[t]{2}{*}{ Strain } & \multicolumn{4}{|c|}{ Insecticide susceptibility } & \multicolumn{4}{|c|}{ Elevated enzyme activity } \\
\hline & prallethrin $+\mathrm{PBO}$ & dimefluthrin & prallethrin & d-allethrin & $\alpha$-EST & $\beta$-EST & GST & MFO \\
\hline Sabak Bernam & $\mathrm{R}$ & M & $\mathrm{R}$ & M & + & + & + & + \\
\hline Kuala Selangor & M & M & $\mathrm{R}$ & S & + & + & + & + \\
\hline Hulu Selangor & $\mathrm{R}$ & $\mathrm{R}$ & M & M & - & - & + & + \\
\hline Gombak & $\mathrm{M}$ & M & M & $\mathrm{S}$ & + & + & - & + \\
\hline Petaling & $\mathrm{s}$ & M & M & $\mathrm{s}$ & + & + & - & - \\
\hline Hulu Langat & M & $\mathrm{R}$ & $\mathrm{R}$ & M & + & + & + & + \\
\hline Kuala Langat & $\mathrm{R}$ & $\mathrm{R}$ & $\mathrm{R}$ & M & + & + & + & + \\
\hline Klang & $\mathrm{R}$ & $\mathrm{R}$ & M & $\mathrm{R}$ & - & - & + & + \\
\hline Sepang & $\mathrm{M}$ & $\mathrm{s}$ & M & $\mathrm{s}$ & + & + & + & + \\
\hline
\end{tabular}

* prallethrin $15.0 \mathrm{mg} / \mathrm{mat}$ with piperonyl butoxide $18.0 \mathrm{mg} / \mathrm{mat}$, dimefluthrin $7.4 \mathrm{mg} / \mathrm{mat}$, prallethrin $15.0 \mathrm{mg} / \mathrm{mat}, \mathrm{d}$-allethrin $40.0 \mathrm{mg} / \mathrm{mat}, \alpha$-EST

$=\alpha$-esterases, $\beta$-EST $=\beta$-esterases, MFO = mixed function oxidases, GST = glutathione-S-transferase, $\mathrm{R}=$ resistant, $\mathrm{M}=$ moderate resistant, $\mathrm{S}=$ susceptible,

$+=$ presence of mechanism, $-=$ absence of mechanism. 
The results showed that pyrethroid-resistance was found in all of the targeted populations, suggesting that pyrethroid has been applied in the study areas for a lengthy period. These area have been affected by dengue outbreak in recent years as highlighted by Leong et al. (2018, 2019). The finding implied the existence of a regional extension of the population where resistant mosquitoes might move away from previous dengue hotspots. Dimefluthrin, as observed in this study, might stimulate some knockdown activity, thus has higher effectiveness compared to prallethrin with PBO, prallethrin and d-allethrin. It has been experimentally demonstrated that dimefluthrin could have faster knockdown activity against $C_{x}$. pipiens pallens and $C_{x}$. quinquefasciatus compared to d-allethrin (Mori, 2017).

In this study, the mosquito mat vaporizer showed effectiveness with high knockdown across all populations tested except Petaling. A lack of efficacy shown in Petaling population is expected as insecticide resistance of Ae. aegypti was reported in this area (Besar et al., 2019). There were comparative differences between knockdown and mortality rate in this study where $d$-allethrin recorded the highest mortality rate which was comparable to the finding by Kudom (2020). In the present study, mat vaporizer containing d-allethrin was found to be high insecticidal activity for Ae. aegypti.

The percentage on knockdown rate of Ae. aegypti to prallethrin with $\mathrm{PBO}$ was higher compared to prallethrin, suggesting $\mathrm{PBO}$ could provide enhanced protection at this concentration. Previous studies in different parts of the world showed the efficacy of PBO in the management of insecticide resistant vectors (Bingham et al., 2011; Fagbohun et al., 2020; Kasai et al., 2014). However, after 60 minutes of exposure, the mortality rate of prallethrin with $\mathrm{PBO}$ decreased in Ae. aegypti, nearly similar with mortality rate of prallethrin. When this point reached, such resistant mosquitoes were predicted to be survived. Likewise, similar results were also reported in Rasli et al. (2021). Therefore, those populations characterised with high or moderate MFO enzyme activities with less or no impact of PBO along with insecticide pyrethroids, should be further investigated, especially on the significance of $k d r$ gene in the development of insecticide resistance.

There were significant associations between knockdown rates of the active ingredients, suggesting cross-resistance in pyrethroid. The cross-resistance arises as those mechanisms overlap due to insecticide strain (Kawada, 2009). Presumably, single pyrethroid insecticide tolerance was suspected of causing cross-resistance to other insecticides in specific class (Du et al., 2016). Significant correlations between prallethrin with $\mathrm{PBO}$ and dimefluthrin, prallethrin with $\mathrm{PBO}$ prallethrin and d-allethrin as well as dimefluthrin and $d$-allethrin were found from the present study. Detection of cross-resistance in pyrethroid in Ae. aegypti was not only confined to Malaysia (Chin et al., 2017), as it was also reported in many countries, i.e., Colombia (Ocampo et al., 2011; Aponte et al., 2018), Mexico (Flores et al., 2013), Thailand (Yaicharoen et al., 2005) and Indonesia (Amelia-Yap et al., 2018a). The relentless incidence of cross-resistance in mosquito mat vaporizer tested might help the local authorities to review the effectiveness of mat vaporizer in the control of mosquitoes.

The recent formulations of the new pyrethroid group of insecticides are d-allethrin, prallethrin, dimefluthrin and metofluthrin (Mori, 2017). Other available household insecticide products apart from mosquito mat vaporizers such as liquid vaporizers, aerosols and coils were widely commercialized, and they were easily accessible in
Malaysian markets. The current study showed that most Ae. aegypti populations were resistant to mosquito mat vaporizers. It may be due to the endophilic nature of $A e$. aegypti, which makes it prone to be subjected to, or in touch with, the chemical produced by these materials and build resistance by selection pressure (Carvalho \& Moreira, 2017).

This study showed the need to alternate various chemicals such as metofluthrin, transfluthrin or $d$ transallethrin in specific locations. The use of pyrethroids in Ae. aegypti pyrethroid-resistant areas should be monitored by follow up studies and management practices should be amended. The results presented may lead to the evaluation of the susceptibility data to be referred by local authorities in determining effective vector control program. There is a possibility that such chemicals may not yield optimal mortality responses for all strains for end-user as the bioassays were carried out under experimental conditions. Hence it is recommended that a semi-field trial at the natural end-user setting to be conducted in future.

Meanwhile, the enzyme assays revealed that only some detoxifying enzymes (i.e., ESTs, GSTs and MFOs) were expressed in pyrethroid-resistant Ae. aegypti. Earlier researches reported the involvement of these enzymes in the contribution of pyrethroid resistance in wild Ae. aegypti (Leong et al., 2019; Pinto et al., 2019; Wan-Norafikah et al., 2010). The variation may indicate that there were multiple resistance mechanisms in Ae. aegypti.

Various studies have shown that the increased EST activity generally resulted from pyrethroid-resistant $A e$. aegypti (Lin et al., 2013; Koou et al., 2014b; Rasli et al., 2018) and $C x$. quinquefasciatus Say (Diptera: Culicidae) (Sarkar et al., 2009; Singh \& Prakash, 2009; Low et al., 2013b; Ramkumar \& Shivakumar, 2015). Their results were inconsistent with the present study, which did not reveal any correlation related to the survivability rate of all insecticides analyzed against $\alpha$-EST activity. However, some pyrethroidsusceptible populations of the field strain demonstrated higher enzyme levels compared to the reference strain. Dichlorodiphenyltrichloroethane (DDT) resistance may have contributed to this detoxification activity (Maestre-Serrano et al., 2014), but this hypothesis is yet to be verified.

Additionally, this study revealed a significant association between the survivability rate of pyrethroids in mosquito vaporizing mat bioassays and GST in Ae. aegypti. There were concerns that this enzyme might not be prevalent due to pyrethroid resistance. Reasonably, the GST enzyme documented the lowest levels in contrast to other groups of enzymes, including those found in the resistant populations. The mean enzyme activities of GST was inversely correlated to the 24-h percentage mortality of Ae. aegypti to prallethrin with $\mathrm{PBO}$ and prallethrin, indicating lower mortality rate with increasing activities of GST in this study. Hemingway \& Ranson (2000) and Ishak et al. (2017) reported that higher rates of GST activity were typically correlated with the exposure to multiple insecticide classes within a large kind of arthropods, primarily due to DDT resistance.

Previous studies also attempted to identify the mechanism of GSTs in DDT resistance in Anopheles gambie and An. funestus (Matiya et al., 2019), An. maculatus (Rohani et al., 2019), Ae. aegypti (Aponte et al., 2018) and CX. quinquefasciatus (Lee \& Chong, 1995; Corbel et al., 2007; Sarkar et al., 2009; Low et al., 2013a, 2013b). To date, associations between insecticide resistance and GST enzyme activity have not been fully identified in a variety of mosquito species worldwide (Amelia-Yap et al., 2019). The detectable GST activity might be owing to the use of pyrethroids in mosquito 
control activities as both DDT and pyrethroids intended to target the voltage-gated sodium channel of arthropods (Amelia-Yap et al., 2018b, 2019; Hemingway \& Ranson, 2000; Koou et al., 2014b). Thus, it assumed that pyrethroid-resistant identified in this study was related to the metabolic detoxification or/and target-site insensitivity. Nevertheless, as mentioned above, the role of GSTs has been restricted in most of the populations with low enzyme activities. Therefore, the used of DDT diagnostic doses of WHO adult bioassay would be recommended in order to explain the significant increased in the production of GST in the studied populations.

Meanwhile, the enzyme assay indicated an increasing level of MFO in Ae. aegypti populations, suggesting MFOs as primary enzymes that stimulated the pyrethroid resistance. Increased levels of MFOs in Ae. aegypti related to pyrethroids resistance were reported elsewhere (Maestre-Serrano et al., 2014; Rasli et al., 2018; Triana et al., 2019; Amelia-Yap et al., 2019). Nonetheless, the increased MFO activity in most mosquito populations might reduce the efficacy of insecticides. The MFOs enzymes are most commonly correlated with cross-resistance between pyrethroids and organophosphates (Pethuan et al., 2007) and DDT (Ngoagouni et al., 2016). Notably, this emphasized the value to evaluate the organophosphate and DDT resistance status in these populations in the years ahead.

A higher RR of the active ingredients tested did not show a consistent activity profile in all enzyme groups, indicating complexity between pyrethroids and enzymes. Thus, metabolic detoxification could not fully explain pyrethroid's elevated resistance status. Several point mutations have been recognized such as F1534C, V1016 G and S989P, homozygous mutations V1016G / S989P (double allele) and F1534C / V1016G / S989P (triple allele) in various dengue vector populations (Leong et al., 2019). Inevitable factors for higher pyrethroid-resistant in wild Ae. aegypti, e.g. behavioural inhibition, cuticle tolerance or target-site insensitivity, were also anticipated (Amelia-Yap et al., 2018b). Further research on synergists would give valuable information on mechanisms of metabolic-mediated resistance.

\section{CONCLUSION}

In conclusion, majority of Ae. aegypti populations in this study have developed resistance to mosquito mat vaporizer containing pyrethroids. This result revealed that pyrethroid resistance has thrived in this country due to the high dependence on vector control. This study also provided reference data and underlined the need for detailed studies on metabolic resistance in Ae. aegypti. In future, resistance might gradually build-up on the susceptible populations if the same control approach was used. Thus, this result urgently suggests reconstructing the national vector control programme in order to monitor the efficacy of pyrethroid against Ae. aegypti.

\section{ACKNOWLEDGEMENTS}

This study was financially supported by the Higher Institution Centre of Excellence (HICOE) program (MO002-2019) and University of Malaya research grant (RP021C/16SUS).

\section{Conflict of interest}

The authors declare there is no conflict of interest.

\section{REFERENCES}

Adanan, C.R., Zairi, J. \& Ng, K.H. (2005). Efficacy and sublethal effects of mosquito mats on Aedes aegypti and Culex quinquefasciatus (Diptera: Culicidae). In: Lee C. Y., Robinson W. H. (editors), Proceedings of The Fifth International Conference on Urban Pests. P \& Y Design Network, Perniagaan Ph'ng, Malaysia. pp: 265-269.

Amelia-Yap, Z.H., Chen, C.D., Sofian-Azirun, M., Lau, K.W., Suana, I.W., Harmonis, Syahputra, E., Razak, A. \& Low, V.L. (2018a). Efficacy of mosquito coils: Cross-resistance to pyrethroids in Aedes aegypti (Diptera: Culicidae) from Indonesia. Journal of Economic Entomology 111: 2854-2860. http:// doi.org/10.1093/jee/toy296

Amelia-Yap, Z.H., Chen, C.D., Sofian-Azirun, M. \& Low, V.L. (2018b). Pyrethroid resistance in the dengue vector Aedes aegypti in Southeast Asia: Present situation and prospects for management. Parasites \& Vectors 11: 332. https:// doi.org/10.1186/s13071-018-2899-0

Amelia-Yap, Z.H., Sofian-Azirun, M., Chen, C.D., Suana, I.W., Lau, K.W., Elia-Amira, N.M.R., Haziqah-Rashid, A., Tan, T.K., Lim, Y.A.L. \& Low, V.L. (2019). Pyrethroids use: Threats on metabolic-mediated resistance mechanisms in the primary dengue vector Aedes aegypti (Diptera: Culicidae). Journal of Medical Entomology 56: 811-816. https://doi.org/ 10.1093/jme/tjz007

Aponte, A., Penilla, R.P.P., Rodriguez, A.D. \& Ocampo, C.B. (2018). Mechanism of pyrethroid resistance in Aedes (stegomyia) aegypti from Colombia. Acta Tropica 191: 146-154. https:// doi.org/10.1016/j.actatropica.2018.12.021

Besar, N.A.U.A., Azman, S., Asri, L.-N. \& Khairuddin, K. (2019). Resistance status of Aedes aegypti towards different insecticides in selected dengue outbreak area in Petaling District (Diptera: Culicinae). Serangga 24: 41-48.

Bibbs, C.S. \& Kaufman, P.E. (2017). Volatile pyrethroids as a potential mosquito abatement tool: A review of pyrethroid-containing spatial repellents. Journal of Integrated Pest Management 8: 1-10. https://doi.org/10. 1093/jipm/pmx016

Bingham, G., Strode, C., Tran, L., Khoa, P.T. \& Jamet, H.P. (2011). Can piperonyl butoxide enhance the efficacy of pyrethroids against pyrethroid-resistant Aedes aegypti? Tropical Medicine and International Health 16: 492-500. https://doi.org/10.1111/j.1365-3156.2010.02717.x

Bisset, J., Rodriguez, M., Soca, A., Pasteur, N. \& Raymond, M. (1997). Cross resistance to pyrethroid and organophosphorus insecticides in the southern house mosquito (Diptera: Culicidae) from Cuba. Journal of Medical Entomology 34: 244-246. https://doi.org/10.1093/jmedent/ 34.2.244

Bong, L.J. \& Zairi, J. (2010). Temporal fluctuations of insecticides resistance in Musca domestica Linn (Diptera: Muscidae) in Malaysia. Tropical Biomedicine 27: 317-325.

Brogdon, W.G., Hobbs, J.H., St Jean, Y., Jacques, J.R. \& Charles, L.B. (1988). Microplate assay analysis of reduced fenitrothion susceptibility in Haitian Anopheles albimanus. Journal of The American Mosquito Control Association 4: 152-158.

Brogdon, W.G., Mcallister, J.C. \& Vulule, J. (1997). Heme peroxidase activity measured in single mosquitoes identifies individuals expressing an elevated oxidase for insecticide resistance. Journal of The American Mosquito Control Association 13: 233-237.

Carvalho, F. \& Moreira, L. (2017). Why is Aedes aegypti Linnaeus so successful as a species? Neotropical Entomology 46: 243-255. https://doi.org/10.1007/s13744-017-0520-4 
Chadwick, P.R. \& Lord, C.J. (1977). Tests of pyrethroid vaporising mats against Aedes aegypti (L.) (Diptera: Culicidae). Bulletin of Entomological Research 67: 667-674. https://doi.org/ 10.1017/S0007485300007033

Chareonviriyaphap, T., Bangs, M.J., Suwonkerd, W., Kongmee, M., Corbel, V. \& Ngoen-Klan, R. (2013). Review of insecticide resistance and behavioral avoidance of vectors of human disease in Thailand. Parasites \& Vectors 6: 280. https://doi.org/10.1186/1756-3305-6-280

Chin, A.C., Chen, C.D., Low, V.L., Lee H.L., Azidah, A.A., Lau, K.W. \& Sofian-Azirun, M. (2017). Comparative efficacy of commercial mosquito coils against Aedes aegypti (Diptera: Culicidae) in Malaysia: A nationwide report. Journal of Medical Entomology 110: 2247-2251. https://doi.org/10.1093/ jee/tox183

Chuaycharoensuk, T., Juntarajumnong, W., Boonyuan, W., Bangs, M.J., Akratanakul, P., Thammapalo, S. \& Chareonviriyaphap, T. (2011). Frequency of pyrethroid resistance in Aedes aegypti and Aedes abopictus (Diptera: Culicidae) in Thailand. Journal of Vector Ecology 36: 204212. https://doi.org/10.1111/j.1948-7134.2011.00158.x

Corbel, V., N'guessan, R., Brengues, C., Chandre, F., Djogbenou, L., Martin, T., Akogbeto, M., Hougard, J.M. \& Rowland, M. (2007). Multiple insecticide resistance mechanisms in Anopheles gambiae and Culex quinquefasciatus from Benin, West Africa. Acta Tropica 101: 207-216. https://doi.org/ 10.1016/j.actatropica.2007.01.005

Du, Y., Nomura, Y., Zhorov, B.S. \& Dong, K. (2016). Sodium channel mutations and pyrethroid resistance in Aedes aegypti. Insects 7: 60. https://doi.org/10.3390/ insects 7040060

El-Garj, F.M.A., Avicor, S.W., Wajidi, M.F.F. \& Jaal, Z. (2015). Comparative efficacy of spatial repellents containing $d$ allethrin and d-trans allethrin against the major dengue vector Aedes aegypti (Linnaeus). Asian Biomedicine 9: 313320. https://doi.org/10.5372/1905-7415.0903.399

Fagbohun, I.K., Idowu, E.T., Olakiigbe, A.K., Oyeniyi, A.T., Otubanjo, O.A. \& Awolola, T.S. (2020). Metabolic resistance mechanism in Aedes aegypti from Lagos State, Nigeria. The Journal of Basic and Applied Zoology 81: 59. https:// doi.org/10.1186/s41936-020-00194-8

Finney, D.J. (1971). Probit analysis. Cambridge: Cambridge University Press.

Flores, A.E., Ponce, G., Silva, B.G., Gutierrez, S.M., Bobadilla, C., Lopez, B., Mercado, R. \& Black, W.C. (2013). Wide spread cross resistance to pyrethroids in Aedes aegypti (Diptera: Culicidae) from Veracruz State Mexico. Journal of Medical Entomology 106: 959-969. https://doi.org/10.1603/ec12284

Hamid, P.H., Ninditya, V.I., Prastowo, J., Haryanto, A., Taubert, A. \& Hermosilla, C. (2018). Current status of Aedes aegypti insecticide resistance development from Banjarmasin, Kalimantan, Indonesia. BioMedical Research International 2018: 1735358. https://doi.org/10.1155/2018/1735358

Hamid, P.H., Prastowo, J., Ghiffari, A., Taubert, A. \& Hermosilla, C. (2017). Aedes aegypti resistance development to commonly used insecticides in Jakarta, Indonesia. PLOS One 12: e0189680. https://doi.org/10.1371/journal.pone. 0189680

Haziqah-Rashid, A., Chen, C.D., Lau, K.W., Low, V.L., SofianAzirun, M., Suana, I.W., Harmonis, H., Syahputra, E., Razak, A., Chin, A.C. et al. (2019). Monitoring insecticide resistance profiles of Aedes aegypti (Diptera: Culicidae) in the Sunda Islands of Indonesia based on diagnostic doses of larvicides. Journal of Medical Entomology 56: 514-518. https://doi.org/10.1093/jme/tjy208
Hemingway, J. \& Ranson, H. (2000). Insecticide resistance in insect vectors of human disease. Annual Review of Entomology 45: 371-391. https://doi.org/10.1146/annurev. ento.45.1.371

Ishak, I.H., Kamgang, B., Ibrahim, S.S., Riveron, J.M., Irving, H. \& Wondji, C.S. (2017). Pyrethroid resistance in Malaysian populations of dengue vector Aedes aegypti is mediated by CYP9 Family of cytochrome P450 genes. PLoS Neglected Tropical Disease 11: e0005302. https://doi.org/10.1371/ journal.pntd.0005302

Jantan, I., Zaki, Z.M., Ahmad, A.R. \& Ahmad R. (1999). Evaluation of smoke from mosquito coils containing Malaysian plants against Aedes aegypti. Fitoterapia 70: 237-243. https://doi.org/10.1016/S0367-326X(99)00026-X

Kasai, S., Komagata, O., Itokawa, K., Shono, T., Ng, L.C., Kobayashi, M. \& Tomita, T. (2014). Mechanisms of pyrethroid resistance in the dengue mosquito vector, Aedes aegypti: Target site insensitivity, penetration, and metabolism. PLoS Neglected Tropical Diseases 8: e0002948. https://doi.org/ 10.1371/journal.pntd.0002948

Katsuda, Y., Leemingsawat, S., Thongrungkiat, S., Prummonkol, S., Samung, Y., Kanzaki, T., Watanabe, T. \& Kahara, T. (2008). Control of mosquito vectors of tropical infectious diseases: (2) Pyrethroid susceptibility of Aedes aegypti (L.) collected from different sites in Thailand. Southeast Asian Journal of Tropical Medicine and Public Health 39: 229234.

Kawada, H. (2009). An inconvenient truth of pyrethroid. Does it have a promising future?. In American Chemical Society Symposium Series 1014: 171-190. https://doi.org/10.1021/bk2009-1014.ch013

Koou, S.-Y., Chong, C.-S., Vythilingam, I., Ng, L.-C. \& Lee, C.-Y. (2014a). Pyrethroid resistance in Aedes aegypti larvae (Diptera: Culicidae) from Singapore. Journal of Medical Entomology 51: 170-181. https://doi.org/10.1603/me13113

Koou, S.-Y., Chong, C.-S., Vythilingam, I., Lee, C.-Y. \& Ng, L.-C. (2014b). Insecticide resistance and its underlying mechanisms in field populations of Aedes aegypti adults (Diptera: Culicidae) in Singapore. Parasites \& Vectors 7: 471. https://doi.org/10.1186/s13071-014-0471-0

Kudom, A.A. (2020). Entomological surveillance to assess potential outbreak of Aedes-borne arboviruses and insecticide resistance status of Aedes aegypti from Cape Coast, Ghana. Acta Tropica 202: 105257. https://doi.org/ 10.1016/j.actatropica.2019.105257

Lee, H.L. (1990). A rapid and simple biochemical method for the detection of insecticide resistance due to elevated esterase activity in Culex quinquefasciatus. Tropical Biomedicine 7: 21-28.

Lee, H.L. (1992). Aedes ovitrap and larval survey in several suburban communities in Selangor, Malaysia. Mosquito Borne Disease Bulletin 9: 9-15.

Lee, H.L. \& Chong, W.L. (1995). Glutathion S-transferase activity and DDT-susceptibility of Malaysian mosquitos. Southeast Asian Journal of Tropical Medicine and Public Health 26: 164167.

Lee, C.Y., Yap, H.H., Chong, N.L. \& Lee, R.S.T. (1996). Insecticide resistance and synergism in field collected German cockroaches (Dictyoptera: Blattellidae) in Peninsular Malaysia. Bulletin of Entomological Research 86: 675-682. https://doi.org/10.1017/s0007485300039195

Lee, R.M.L., Choong, C.T.H., Goh, B.P.L, Ng, L.C. \& Lam-Phua, S.G. (2014). Bioassay and biochemical studies of the status of pirimiphos-methyl and cypermethrin resistance in Aedes (Stegomyia) aegypti and Aedes (Stegomyia) albopictus (Diptera: Culicidae) in Singapore. Tropical Biomedicine 31: 670-679. 
Leong, C.S., Vythilingam, I., Liew, J.W.K., Wong, M.L., Wan Sulaiman, W.Y. \& Lau, Y.L. (2019). Enzymatic and molecular characterization of insecticide resistance mechanisms in field populations of Aedes aegypti from Selangor, Malaysia. Parasites \& Vectors 12: 236. https://doi.org/ 10.1186/s13071-019-3472-1

Leong, C.S., Vythilingam, I., Wong, M.L., Wan Sulaiman, W.-Y. \& Lau, Y.L. (2018). Aedes aegypti (Linnaeus) larvae from dengue outbreak areas in Selangor showing resistance to pyrethroids but susceptible to organophosphates. Acta Tropica 185: 115-126. https://doi.org/10.1016/j.actatropica. 2018.05.008

Lin, Y.H., Tsen, W.L., Tien, N.Y. \& Luo, Y.P. (2013). Biochemical and molecular analyses to determine pyrethroid resistance in Aedes aegypti. Pesticide Biochemistry and Physiology 107: 266-276. https://doi.org/10.1016/j.pestbp.2013.08.004

Liu, W., Zhang, J., Hashim, J.H., Jalaludin, J., Hashim, Z. \& Goldstein, B.D. (2003). Mosquito coil emissions and health implications. Environmental Health Perspectives 111: 1454-1460. https://doi.org/10.1289/ehp.6286

Low, V.L., Chen, C.D., Lee, H.L., Lim, P.E., Leong, C.S. \& SofianAzirunm M. (2013a). Current susceptibility status of Malaysian Culex quinquefasciatus (Diptera: Culicidae) against DDT, propoxur, malathion and permethrin. Journal of Medical Entomology 50: 103-111. https://doi.org/10.1603/ ME12068

Low, V.L., Chen, C.D., Lee, H.L., Tan, T.K., Chen, C.F., Leong, C.S., Lim, Y.A.L., Lim, P.E., Norma-Rashid, Y. \& Sofian-Azirun, M. (2013b). Enzymatic characterization of insecticide resistance mechanisms in field populations of Malaysian Culex quinquefasciatus say (Diptera: Culicidae). PLoS One 8: e79928. https://doi.org/10.1371/journal.pone.0079928

Maestre-Serrano, R., Gomez-Camargo, D., Ponce-Garcia, G. \& Flores, A.E. (2014). Susceptibility to insecticides and resistance mechanisms in Aedes aegypti from the Colombian Caribbean Region. Pesticide Biochemistry and Physiology 116: 63-73. https://doi.org/10.1016/j.pestbp. 2014.09.014

Matiya, D.J., Philbert, A.B., Kidima, W. \& Matowo, J.J. (2019). Dynamics and monitoring of insecticide resistance in malaria vectors across mainland Tanzania from 1997 to 2017: A systematic review. Malaria Journal 18: 102. https:// doi.org/10.1186/s12936-019-2738-6

Mazzarri, M.B. \& Georghiou, G.P. (1995). Characterization of resistance to organophosphate, carbamate and pyrethroid insecticides in field populations of Aedes aegypti from Venezuela. Journal of The American Mosquito Control Association 11: 315-322.

(MOH) Ministry of Health Malaysia (2009). Pelan strategik pencegahan dan kawalan denggi 2009-2013. https:// www.moh.gov.my/moh/resources/auto\%20download \%20images/589d71fa1d2b5.pdf. Accessed 10 January 2019.

(MOH) Ministry of Health Malaysia (2020). Kenyataan Akhbar Khas Ketua Pengarah Kesihatan Malaysia situasi semasa demam denggi, zika dan chikungunya di Malaysia dan Inisiatif KKM untuk memerangi denggi Tahun 2020. http://www.myhealth.gov.my/wp-content/uploads/ Kenyataan-Akhbar-Khas-Ketua-Pengarah-KesihatanMalaysia-2020.pdf. Accessed 10 January 2020.

Mori, T. (2017). Recent Findings of New Synthetic Pyrethroids. Acta Hortic 47-52. https://doi.org/10.17660/ActaHortic.2017. 1169.8

Ngoagouni, C., Kamgang, B., Brengues, C., Yahouedo, G., Paupy C., Nakoune, E., Kazanji, M. \& Chandre, F. (2016). Susceptibility profile and metabolic mechanisms involved in Aedes aegypti and Aedes albopictus resistant to DDT and deltamethrin in the Central African Republic. Parasites \& Vectors 9: 599. https://doi.org/10.1186/s13071-016-1887-5

Ocampo, C.B., Salazar-Terreros, M.J., Mina, N.J., Mcallister, J. \& Brogdon, W. (2011). Insecticide resistance status of Aedes aegypti in 10 localities in Colombia. Acta Tropica 118: 37-44. https://doi.org/10.1016/j.actatropica.2011.01. 007

Pethuan, S., Jirakanjanakit, N., Saengtharatip, S., Chareonviriyaphap, T., Kaewpa, D. \& Rongnoparut, P. (2007). Biochemical studies of insecticide resistance in Aedes (Stegomyia) aegypti and Aedes (Stegomyia) albopictus (Diptera: Culicidae) in Thailand. Tropical Biomedicine 24: 7-15.

Pinto, J., Palomino, M., Mendozoa-Uribe, L., Sinti, C., Liebman, K.A. \& Lenhart, A. (2019). Susceptibility to insecticides and resistance mechanisms in three populations of Aedes aegypti from Peru. Parasites \& Vectors 12: 494. https:// doi.org/10.1186/s13071-019-3739-6

Ramkumar, G. \& Shivakumar, M.S. (2015). Laboratory development of permethrin resistance and cross-resistance pattern of Culex quinquefasciatus to other insecticides. Parasitology Research 114: 2553-2560. https://doi.org/ 10.1007/s00436-015-4459-2

Rasli, R., Lee, H.L., Ahmad, N.W., Fikri, S.F.F., Ali, R., Muhamed, K.A., Hadi, A.A., Liu, Q.-Y. \& Meng, F.X. (2018). Susceptibility status and resistance mechanisms in permethrinselected, laboratory susceptible and field-collected Aedes aegypti from Malaysia. Insects 9: 43. https://doi.org/ $10.3390 /$ insects 9020043

Rasli, R., Cheong, Y.L., Ibrahim, M.K.C, Fikri, S.F.F., Norzali, R.N., Nazarudin, N.A., Fikri, S.F.F, Norzali, R.N., Nazarudin N.A., Hamdan, F.N. et al. (2021). Insecticide resistance in dengue vectors from hotspots in Selangor, Malaysia. PLoS Neglected Tropical Diseases 15: e0009205. https://doi.org/10.1371/ journal.pntd.0009205

Rohani, A., Azahary, A.R.A., Yu, K., Zurainee, M.N., Wan Najdah, W.M.A. \& Lee, H.L. (2019). Insecticide susceptibility status and resistance mechanism of Anopheles cracens Sallum and Peyton and Anopheles maculatus Theobald (Family: Culicidae) from knowlesi malaria endemic areas in Peninsular Malaysia. Asian Pacific Journal of Tropical Medicine 12: 79-86. https://doi.org/10.4103/1995-7645.250841

Sarkar, M., Bhattacharyya, I.K., Borkotoki, A., Goswami, D., Rabha, B., Baruah, I. \& Srivastava, R.B. (2009). Insecticide resistance and detoxifying enzyme activity in the principal bancroftian filariasis vector, Culex quinquefasciatus, in Northeastern India. Medical and Veterinary Entomology 23: 122-131. https://doi.org/10.1111/j.1365-2915.2009.00805.x

Sathantriphop, S., Onkong, S., Paeporn, P., Ya-Umphan, P., Mukkhun, P., Bangs, M.J. \& Kongmee, M. (2019). Knockdown and lethal effects of three mosquito coil formulations against Aedes aegypti and Culex quinquefasciatus under different nutritional conditions. Journal of Asia-Pacific Entomology 22: 1046-1052. https://doi.org/10.1016/j.aspen. 2019.08.007

Sathantriphop, S., Paeporn, P., Ya-Umphan, P., Mukkhun, P., Thanispong, K., Chansang, C., Bangs, M.J., Chareonviriyaphap, T. \& Tainchum, K. (2020). Behavioral action of deltamethrin and cypermethrin in pyrethroid-resistant Aedes aegypti (Diptera: Culicidae): Implications for control strategies in Thailand. Journal of Medical Entomology 57: 1157-1167. https://doi.org/10.1093/jme/tjaa019

Sayono, S., Mudawamah, P.L., Meikawati, W. \& Sumanto, D. (2019). Effect of d-allethrin aerosol and coil to the mortality of mosquitoes. Journal of Arthropod-Borne Disease 13: 259-267. https://doi.org/10.18502/jad.v13i3.1536 
Singh, G. \& Prakash, S. (2009). Gokilaht ${ }^{\circledR}$-S 5EC testing on Culex quinquefasciatus Say larvae for an early detection in esterase and monooxygenase resistance system. Parasitology Research 104: 1087-1091. https://doi.org/10. 1007/s00436-008-1292-x

Triana, D., Umniyati, S.R. \& Mulyaningsih, B. (2019). Resistance status of Aedes aegypti to malathion and cypermethrin in Bengkulu City, Indonesia. Southeast Asian Journal Tropical Medical Public Health 50: 1-8.

Vontas, J., Kioulos, E., Pavlidi, N., Morou, E., Della Torre, A. \& Ranson, H. (2012). Insecticide resistance in the major dengue vectors Aedes albopictus and Aedes aegypti. Pesticide Biochemistry and Physiology 104: 126-131. https://doi.org/ 10.1016/j.pestbp.2012.05.008

Wan-Norafikah, O., Nazni, W.A., Lee, H.L., Zainol-Ariffin, P. \& Sofian-Azirun, M. (2010). Permethrin resistance in Aedes aegypti (Linnaeus) collected from Kuala Lumpur, Malaysia. Journal of Asia-Pacific Entomology 13: 175-182. https:// doi.org/10.1016/j.aspen.2010.03.003
(WHO) World Health Organization (2009). Guidelines for efficacy testing of household insecticide products: mosquito coils, vaporizer mats, liquid vaporizers, ambient emanators and aerosols. Control of Neglected Tropical Diseases, WHO Pesticide Evaluation Scheme. https:// www.who.int/whopes/resources/who_htm_ntd_ whopes_2009.3/en/. Accessed 26 October 2018.

(WHO) World Health Organization (2016). Monitoring and managing insecticide resistance in Aedes mosquito populations: Interim guidance for entomologist. https:// www.who.int/csr/resources/publications/zika/ insecticide-resistance/en/. Accessed 26 October 2018.

Yaicharoen, R., Kiatfuengfoo, R., Chareonviriyaphap, T. \& Rongnoparut, P. (2005). Characterization of deltamethrin resistance in field populations of Aedes aegypti in Thailand. Journal of Vector Ecology 30: 144-150.

Yap, H., Lee, C., Chong, N., Baba, R. \& Tan, H. (1995). Laboratory bioassays of Malaysian standard mosquito mat formulation against Aedes aegypti (L.) and Culex quinquefasciatus (Say) using two test methods. Journal of Biosciences 6: 8693. 